\title{
Doença aguda das vias aéreas inferiores em menores de cinco anos: influência do ambiente doméstico e do tabagismo materno
}

\author{
Acute disease of the lower airways in children under five years of age: \\ role of domestic environment and maternal cigarette smoking \\ Silvio O.M. Prietsch ${ }^{1}$, Gilberto B. Fischer ${ }^{2}$, Juraci A. Cesar ${ }^{3}$, Alisson R. Fabris ${ }^{4}$, \\ Halid Mehanna ${ }^{4}$, Tissyanna H.P. Ferreira ${ }^{4}$, Leopoldo A. Scheifer ${ }^{4}$
}

\section{Resumo}

Objetivos: estudar a prevalência de doença aguda das vias aéreas inferiores e a influência dos fatores relacionados às condições de moradia e do tabagismo materno. Dentre os fatores de risco reconhecidos, tabagismo, aglomeração e condições de moradia desfavoráveis têm papel fundamental na cadeia causal dessas doenças.

Métodos: foi obtida uma amostra de 775 crianças de zero a 59 meses, da cidade do Rio Grande, RS, Brasil, através de estudo transversal, de base populacional. Entrevistadores treinados aplicaram questionário padronizado às mães ou responsáveis pelas crianças em seus domicílios, e coletaram informações sobre características maternas, condições de habitação, nível socioeconômico da família e tabagismo. Os fatores ambientais foram estudados individualmente, $\mathrm{e}$ através de um escore capaz de avaliar a intensidade das associações com doença respiratória. Foram realizadas análises bivariada, com o cálculo das razões de prevalência de cada um dos fatores de risco, e multivariada, através de regressão logística não condicional.

Resultados: estiveram diretamente associados com doença respiratória: ambiente desfavorável $(\mathrm{p}<0,01)$, escolaridade materna menor que cinco anos $(\mathrm{p}=0,01)$, renda familiar menor que US $\$ 200(\mathrm{p}=0,04)$, aglomeração $(p=0,02)$, tabagismo durante a gestação $(p=0,03)$ e tabagismo materno atual $(\mathrm{p}=0,01)$. Idade materna igual ou superior a 30 anos foi identificada como um fator de proteção $(\mathrm{p}=0,05)$.

Conclusões: esses resultados mostram que é necessário melhorar a distribuição da renda, melhorar os índices de escolaridade, melhorar as condições de moradia e combater o hábito de fumar, especialmente entre as mães. Os programas de controle das doenças respiratórias devem contemplar esses pontos críticos, que representam um importante risco para a saúde infantil.

$J$ Pediatr (Rio J) 2002; 78 (5): 415-22: epidemiologia, doença respiratória, sibilância, fatores de risco ambientais, tabagismo passivo.

\begin{abstract}
Objective: to study the prevalence of acute disease of the lower airways and the role of the domestic environment and maternal smoking. Among the recognized risk factors, passive smoking, living in crowded environments and poor housing conditions play a fundamental role in the causal chain of these diseases.

Methods: a cross-sectional study was carried out in a sample of 775 children aged between 0 and 59 months living in Rio Grande, southern Brazil. Trained interviewers applied a standardized questionnaire to the mothers or guardians of these children in their homes and gathered information about maternal characteristics, housing conditions, socio-economic status of the family and smoking habits. Environmental factors were individually studied, and classified according to a score that evaluated the intensity of their association with respiratory diseases. Bivariate analyses were performed, calculating the prevalence ratios for each risk factor, as well as multivariate ones, by means of non-conditional regression analyses.
\end{abstract}

Results: the main risks identified were: unfavorable environment $(P<0.01)$, less than five years of maternal educational level $(P=0.01)$, monthly family income under US $\$ 200(P=0.04)$, crowded environments $(P=0.02)$, smoking during pregnancy $(P=0.03)$ and present maternal smoking $(P=0.01)$. A thirty-year-old or older mother was identified as offering a protection factor $(P=0.05)$.

Conclusions: these results indicate the need to improve the income distribution, improve the rates of educational level, and combat the smoking habit, particularly concerning mothers. The programs of control of respiratory diseases must address these critical points that represent an important risk to children's health.

JPediatr (RioJ) 2002; 78 (5): 415-22: epidemiology, respiratory illnesses, wheeze, environmental risk factors, passive smoking.

1. Prof. Assistente Mestre em Pediatria - Departamento Materno-Infantil. Fundação Universidade Federal de Rio Grande (FURG)

2. Prof. Titular Doutor em Pneumologia - Faculdade Federal de Ciências Médicas de Porto Alegre.

3. Prof. Assistente Mestre em Epidemiologia - Departamento Materno-Infantil. Fundação Universidade Federal de Rio Grande.

4. Aluno do 60 ano de Medicina - Bolsista da FURG.

Artigo submetido em 07.03.02, aceito em 19.06.02. 


\section{Introdução}

As doenças agudas das vias aéreas inferiores são os principais motivos de manutenção das altas taxas de morbimortalidade em menores de cinco anos, nos países em desenvolvimento, responsáveis por mais de 4 milhões de óbitos por ano ${ }^{1,2}$. Dentre os fatores de risco já identificados, as condições ambientais (sazonalidade, aglomeração, poluição atmosférica, poluição doméstica e tabagismo) apresentam papel de destaque na cadeia causal dessas doenças ${ }^{3}$.

A aglomeração é extremamente comum nas famílias das regiões menos desenvolvidas, nas quais a taxa de natalidade é quase sempre muito elevada e as condições de moradia são ruins, inclusive por um limitado número de peças utilizadas por seus habitantes ${ }^{3}$. Especialmente quanto ao número de moradores e ao número de crianças menores de cinco anos no domicílio, existe clara associação com as doenças respiratórias ${ }^{4}$.

$\mathrm{O}$ alto custo e a limitada disponibilidade de eletricidade tornam freqüente o uso de combustíveis orgânicos, como fonte de energia para cozinhar ou aquecer o ambiente, nas comunidades pobres, muitas vezes sem o necessário cuidado com a eliminação da fumaça e de outros resíduos provenientes da combustão ${ }^{5}$. É possível observar, nessa situação, concentrações até 20 vezes maiores de material poluente ${ }^{6}$. A exposição a aeroalérgenos, especialmente ao ácaro do pó doméstico, a componentes da saliva e aos pêlos de animais, tem sido relacionada à resposta inflamatória da mucosa respiratória e ao conseqüente estabelecimento de hiper-reatividade e ocorrência secundária de doença respiratória ${ }^{7}$.

O fumo do tabaco é, no entanto, o principal poluente doméstico $^{8}$. Como a prevalência do hábito de fumar é alta, especialmente nas áreas urbanas dos países menos desenvolvidos, onde cerca de um terço das mulheres e quase a metade dos homens são fumantes, as taxas de exposição ao fumo passivo, para as crianças, estão entre 38 a $45 \%{ }^{9}$.

Este estudo teve por objetivo medir a prevalência e identificar os principais fatores de risco ambientais associados à doença aguda das vias aéreas inferiores, na cidade de Rio Grande, RS, Brasil.

\section{Material e métodos}

Rio Grande possui cerca de 200.000 habitantes, localizada no extremo sul do Brasil, apresenta temperaturas variando de $0^{\circ} \mathrm{C}$ no inverno a $36^{\circ} \mathrm{C}$ no verão. Sua economia está baseada nas atividades pesqueira, petroquímica e portuária ${ }^{10}$.

Foram incluídas crianças de 0 a 59 meses de idade, residentes na zona urbana do município, entre os meses de agosto e novembro de 1997. Para alcançar uma precisão de $5 \%$, nível de confiança de $95 \%$, e poder de $80 \%$, seriam necessárias informações de 771 crianças ${ }^{11}$. A esse valor já foram incluídos $10 \%$ para compensar possíveis perdas, e $15 \%$ para controle de fatores de confusão ${ }^{12}$.
Utilizou-se uma amostragem sistemática, por conglomerados, tendo como base os setores censitários do Instituto Brasileiro de Geografia e Estatística (IBGE). Cada setor censitário é formado por um conjunto de 200 a 300 domicílios já demarcados pelo IBGE. Trabalhamos com um terço desses setores, totalizando 66 setores (198/3). Como os dados do IBGE estimavam haver um menor de cinco anos em cada quatro domicílios, seria necessário visitar 47 domicílios em cada setor $(47 \mathrm{x} 0,25 \mathrm{x} 66)$. Os setores foram colocados em uma lista e numerados, o primeiro foi escolhido aleatoriamente, e os demais foram sistematizados, pulando de três em três na listagem. Cada quarteirão, em cada setor, foi também numerado e sorteado, assim como as suas esquinas, para ter-se o ponto de partida da pesquisa. A partir desse ponto, no sentido horário, visitavam-se 47 domicílios consecutivos, passando ao quarteirão seguinte até atingir o número de domicílios necessário.

Quatorze acadêmicos voluntários dos cursos de Medicina e de Enfermagem da Universidade Federal do Rio Grande foram treinados para aplicar questionários padronizados às mães ou responsáveis pelas crianças, e pesar e medir estatura ou comprimento de menores de cinco anos. Foram utilizados dois questionários, um para as características das mães e famílias e outro sobre a criança. Esses questionários buscavam informações sobre características demográficas e reprodutivas maternas, saneamento e moradia, nível socioeconômico, comprimento ou estatura, e peso atual e ao nascer.

Os fatores ambientais foram estudados individualmente e através de um escore pontuado de zero a dez, criado a partir das variáveis: tipo de construção e de piso, aquecimento doméstico, tipo de fogão, presença de animais domésticos na casa e no quarto da criança, aglomeração e fumo materno. Quanto maior a pontuação nesse escore, piores eram as condições ambientais.

Doença aguda das vias aéreas inferiores foi definida pela ocorrência de tosse e dificuldade respiratória referida (respiração acelerada ou respiração difícil), com ou sem retração subcostal e/ou intercostal, que tenha necessitado de cuidados médicos nos últimos sete dias. Para menores de 12 meses, considerou-se sibilância ou tosse nos últimos sete dias ${ }^{13-15}$. A presença de dois ou mais episódios de sibilância foi critério de exclusão em qualquer faixa etária.

Para entrada dos dados, comparação de digitações e análise de consistência, utilizou-se o programa Epi-Info $6.02^{11}$. No programa SPSS for Windows, versão 8.0, foram feitas as análises bivariada e multivariada ${ }^{16}$.

O plano de análise obedeceu às seguintes etapas: (1) listagem de freqüência de todas variáveis (desfecho, fatores de risco e de confusão) e exame de sua distribuição, assim como das suas medidas de tendência central (média, mediana e moda) e de dispersão (amplitude e desvio-padrão), quando possível; (2) recodificação, com definição de pontos de corte para fatores de risco e de confusão; (3) cruzamento das variáveis independentes com o desfecho. A magnitude das associações foi estimada através da razão de 
prevalências (prevalência nos expostos dividida pela prevalência nos não expostos), e de seu intervalo de confiança de 95\%. Para calcular a significância estatística, foi utilizado o teste do Qui-quadrado $\left(\chi^{2}\right)$ de Pearson, com a correção de Yates para variáveis dicotômicas. Também foi utilizado o teste para tendência linear em proporções nas variáveis categóricas com ordenação lógica; (4) testou-se possíveis associações entre as variáveis independentes que poderiam confundir a associação do desfecho com os fatores de risco; (5) finalmente, efetuou-se análise multivariada, controlando simultaneamente diversos fatores de risco e/ou confusão, através de um modelo de regressão logística não condicional, para obtenção das razões de prevalência, e aplicou-se o teste de razão de verossimilhança, para obtenção da significância estatística ${ }^{17}$. Foram testadas no modelo todas as variáveis que apresentaram, na análise bivariada, um $p$ com valor menor ou igual a $0,20^{18}$.

Essa análise levou em consideração um modelo hierarquizado, no qual algumas variáveis são sobredeterminantes em relação às demais ${ }^{19}$. Nesse modelo, pressupõe-se que o desfecho varia de acordo com os fatores de risco. Embora alguns deles não sejam responsáveis diretos pela ocorrência da doença, potencializam o efeito de outros determinantes. As variáveis demográficas (sexo, cor e idade), embora atuem sobre o desfecho, não são influenciadas por outros fatores, mas podem, a princípio, ser determinantes sobre os demais. O nível socioeconômico exerce influência sobre as características reprodutivas maternas e sobre os fatores ambientais, pois estabelece condições de exposição. Por essa razão, os fatores socioeconômicos foram analisados no primeiro nível, junto com as variáveis demográficas. No segundo nível, estão, portanto, as características maternas e os fatores ambientais. Nesse modelo, atuando junto ou isoladamente, os fatores de risco têm um importante papel no estabelecimento (ou não) de doença aguda das vias aéreas inferiores.

\section{Resultados}

Dentre os 3.185 domicílios visitados, foram identificadas 792 crianças. Destas, 775 foram incluídas no estudo, 13 não foram encontradas, e quatro se recusaram a participar do estudo. Estes dados contabilizaram perdas de 2,1\%.

A distribuição das crianças foi semelhante quanto à faixa etária e ao sexo, mas $83 \%$ tinham cor branca. Metade das famílias possuía cinco ou mais membros, e em um terço havia pelo menos dois menores de cinco anos. Sete por cento das mães e $4 \%$ dos pais não sabiam ler ou escrever. Uma em cada seis famílias possuía renda mensal de até US\$100, e cerca de $30 \%$ tiveram renda mensal de até US\$ 200 . Pouco mais de $70 \%$ delas moravam em casas de tijolos, e cerca de $10 \%$, em cortiços (casas construídas com lata, plástico ou papelão). Praticamente em todos os domicílios havia disponibilidade de água tratada, e $90 \%$ possuíam rede de esgotos. Noventa por cento das famílias cozinhavam com fogão a gás, e 10\% utilizavam fogão a lenha. Setenta e cinco por cento das residências não dispunham de qualquer equipamento para o aquecimento doméstico. Em metade dos domicílios havia duas ou mais pessoas por cômodo, e $66 \%$ deles abrigavam três ou mais pessoas por dormitório. A maioria das famílias tinha animais domésticos, com a predominância de cães, presentes em $62 \%$ dos domicílios. Em $63 \%$ dos domicílios havia pelo menos um fumante (Figura 1). Quarenta por cento dos pais eram fumantes, assim como $37 \%$ das mães. As mães fumavam,

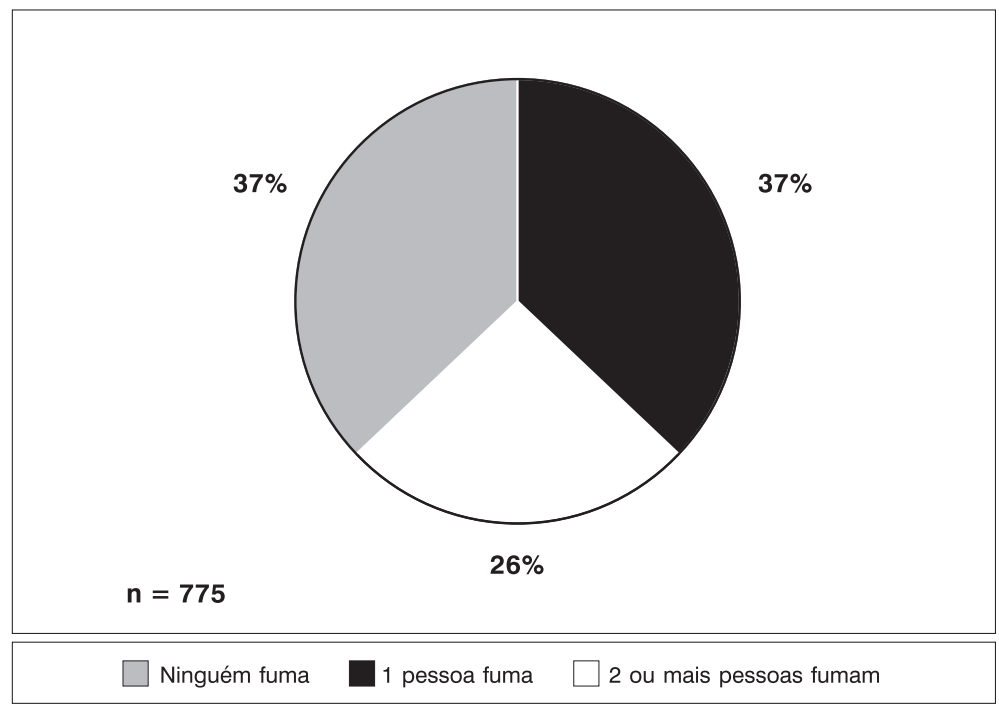

Figura 1 - Percentual de fumantes nos domicílios, Rio Grande, RS, 1997 
em média, 14 cigarros por dia (desvio-padrão de 8,1 ), e os pais, 17 cigarros (desvio-padrão de 9,4).

A prevalência geral de doença aguda das vias aéreas inferiores foi de 23,9\% (IC95\% 21,1 - 26,9). Na análise bivariada, estiveram significativamente associadas às seguintes variáveis: escolaridade materna, escolaridade paterna, renda familiar, idade materna, fumo durante a gravidez, aglomeração (pessoa/peça e pessoa/quarto), fumo materno, número de fumantes no domicílio e escore ambiental desfavorável. A Tabela 1 mostra as variáveis introduzidas no primeiro nível no modelo de regressão logística. Observou-se que o risco de doença respiratória foi $65 \%$ maior para crianças que tinham mães com menor nível de escolaridade, e $50 \%$ maior quando as famílias tiveram renda mensal inferior a US\$ 200.

$\mathrm{Na}$ Tabela 2, observamos as variáveis introduzidas no segundo nível. Ter filhos aos 30 anos ou mais mostrou-se um fator protetor à ocorrência de doença nas crianças, enquanto que a aglomeração familiar e fumo materno apresentaram claramente efeito dose-resposta: quanto maior o número de pessoas no domicílio e maior a quantidade de cigarros fumados pela mãe, maior o risco de doença aguda das vias aéreas inferiores.

Duzentos e vinte e quatro mães (29\%) fumaram, em média, 12,5 cigarros durante a gravidez. A Figura 2 mostra, na análise bivariada, uma tendência linear de associação entre o número de cigarros fumados pelas gestantes e a presença de doença respiratória atual nas crianças.

O escore ambiental foi introduzido no segundo nível, juntamente com a idade materna, substituindo as variáveis do ambiente doméstico. Após ajuste para fatores socioeconômico e idade materna, observou-se associação direta: quanto maior o número de pontos obtidos no escore, maior o risco de doença respiratória. Para aquelas crianças que viviam em presença de cinco ou mais fatores desfavoráveis, o risco foi cerca de duas vezes maior, mesmo após o ajuste para todas as variáveis estudadas (Figura 3 ).

Tabela 1 - Razões de prevalência e teste de razão de verossimilhança (TRV) para doença aguda das vias aéreas inferiores em menores de cinco anos. Análise multivariada por regressão logística, conforme cor da criança e variáveis socioeconômicas, Rio Grande, RS, 1997

\begin{tabular}{|c|c|c|}
\hline \multirow[t]{2}{*}{ Variáveis (modelo hierárquico) } & \multicolumn{2}{|c|}{ Razão de Prevalência (IC 95\%) } \\
\hline & Bruta & Ajustada* \\
\hline \multicolumn{3}{|l|}{ Cor da criança } \\
\hline Branca & 1,00 & 1,00 \\
\hline Mista e negra & $1,29(0,96-1,74)$ & $1,18(0,86-1,61)$ \\
\hline TRV categórico & $\mathrm{p}=0,13$ & $\mathrm{p}=0,30$ \\
\hline \multicolumn{3}{|c|}{ Escolaridade materna (anos completos) } \\
\hline 0 a 4 & $1,70(1,18-2,44)$ & $1,65(1,15-2,37)$ \\
\hline 5 a 8 & $1,49(1,04-2,12)$ & $1,47(1,04-2,08)$ \\
\hline 9 ou mais & 1,00 & 1,00 \\
\hline TRV categórico & $\mathrm{p}=0,01$ & $\mathrm{p}=0,02$ \\
\hline TRV tendência linear & $\mathrm{p}<0,01$ & $\mathrm{p}=0,01$ \\
\hline \multicolumn{3}{|c|}{ Escolaridade paterna (anos completos) } \\
\hline 0 a 4 & $1,54(1,04-2,27)$ & $1,33(0,92-1,92)$ \\
\hline 5 a 8 & $1,45(1,00-2,10)$ & $1,32(0,90-1,93)$ \\
\hline 9 ou mais & 1,00 & 1,00 \\
\hline TRV categórico & $\mathrm{p}=0,06$ & $\mathrm{p}=0,35$ \\
\hline TRV tendência linear & $\mathrm{p}=0,03$ & $\mathrm{p}=0,22$ \\
\hline \multicolumn{3}{|l|}{ Renda familiar (dólares americanos) } \\
\hline Menos de U\$ 200 & $1,73(1,25-2,40)$ & $1,50(1,02-2,20)$ \\
\hline U\$ 200 a U\$ 500 & $1,37(0,99-1,88)$ & $1,23(0,96-1,57)$ \\
\hline Mais de U\$ 500 & 1,00 & 1,00 \\
\hline TRV categórico & $\mathrm{p}<0,01$ & $\mathrm{p}=0,12$ \\
\hline TRV tendência linear & $\mathrm{p}=0,001$ & $\mathrm{p}=0,04$ \\
\hline
\end{tabular}

* Ajustado por cor da pele, escolaridade materna, escolaridade paterna, renda familiar. 
Tabela 2 - Razões de prevalência e teste de razão de verossimilhança (TRV) para doença aguda das vias aéreas inferiores em menores de cinco anos. Análise multivariada, conforme idade materna, aglomeração e tabagismo, Rio Grande, RS, 1997

\begin{tabular}{|c|c|c|}
\hline \multirow[t]{2}{*}{ Variáveis (modelo hierárquico) } & \multicolumn{2}{|c|}{ Razão de Prevalência (IC 95\%) } \\
\hline & Bruta & Ajustada* \\
\hline \multicolumn{3}{|c|}{ Idade materna ao nascimento (em anos) } \\
\hline 13 a 19 & $1,06(0,78-1,43)$ & $1,01(0,87-1,17)$ \\
\hline 20 a 29 & 1,00 & 1,00 \\
\hline 30 ou mais & $0,73(0,53-1,00)$ & $0,70(0,50-0,98)$ \\
\hline TRV categórico & $\mathrm{p}=0,08$ & $\mathrm{p}=0,07$ \\
\hline TRV tendência linear & $\mathrm{p}=0,04$ & $\mathrm{p}=0,05$ \\
\hline \multicolumn{3}{|l|}{ Aglomeração (pessoa/quarto) } \\
\hline 2 ou menos & 1,00 & 1,00 \\
\hline 3 & $1,43(1,03-1.99)$ & $1,33(0,96-1,85)$ \\
\hline 4 ou mais & $1,72(1,23-2,41)$ & $1,54(1,08-2,19)$ \\
\hline TRV categórico & $\mathrm{p}<0,01$ & $\mathrm{p}=0,05$ \\
\hline TRV tendência linear & $\mathrm{p}=0,001$ & $\mathrm{p}=0,02$ \\
\hline \multicolumn{3}{|c|}{ Fumo materno (número de cigarros/dia) } \\
\hline Não fuma & 1,00 & 1,00 \\
\hline 1 a 9 & $1,33(0,89-1,99)$ & $1,22(0,80-1,86)$ \\
\hline 10 ou mais & $1,43(1,09-1,87)$ & $1,41(1,07-1,86)$ \\
\hline TRV categórico & $\mathrm{p}=0,03$ & $\mathrm{p}=0,05$ \\
\hline TRV tendência linear & $\mathrm{p}<0,01$ & $\mathrm{p}=0,01$ \\
\hline \multicolumn{3}{|l|}{ Fumantes no domicílio } \\
\hline Ninguém fuma & 1,00 & 1,00 \\
\hline 1 ou mais & $1,34(1,02-1,77)$ & $1,33(0,99-1,89)$ \\
\hline TRV categórico & $\mathrm{p}=0,04$ & $\mathrm{p}=0,06$ \\
\hline
\end{tabular}

\section{Discussão}

A prevalência de doença aguda das vias aéreas inferiores é bastante elevada na amostra estudada. Além do baixo nível de escolaridade materna, as precárias condições de moradia, aglomeração familiar e o tabagismo materno foram importantes determinantes da ocorrência de doença.
Ao discutir esses resultados, é preciso ter-se em mente que o delineamento que foi utilizado não é o mais apropriado para medir relação de causalidade. O delineamento transversal foi utilizado pela alta representatividade, baixo custo, rapidez, facilidade de execução e, especialmente, por

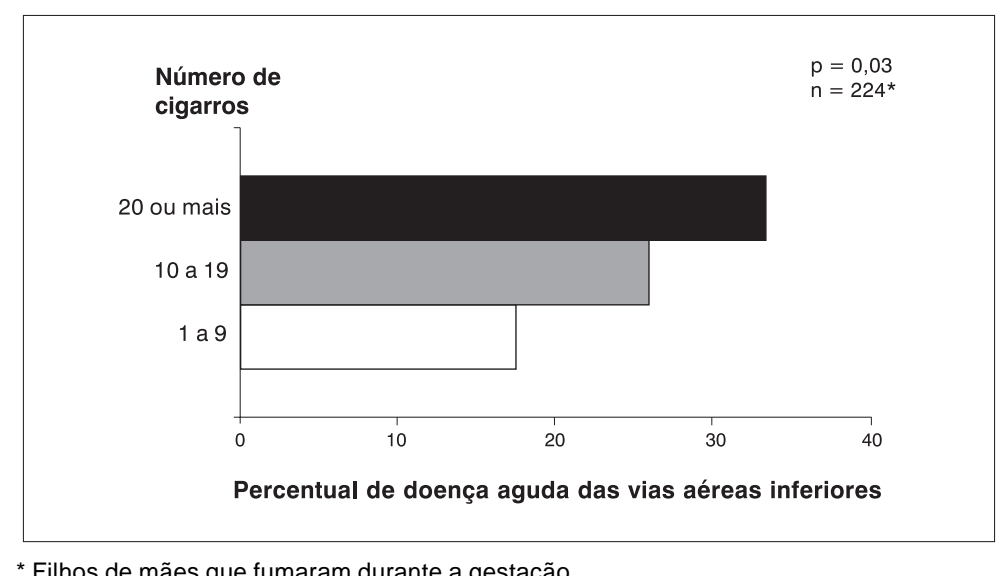

Figura 2 - Percentual de doença aguda das vias aéreas inferiores, conforme o número de cigarros fumados pela mãe durante a gestação 


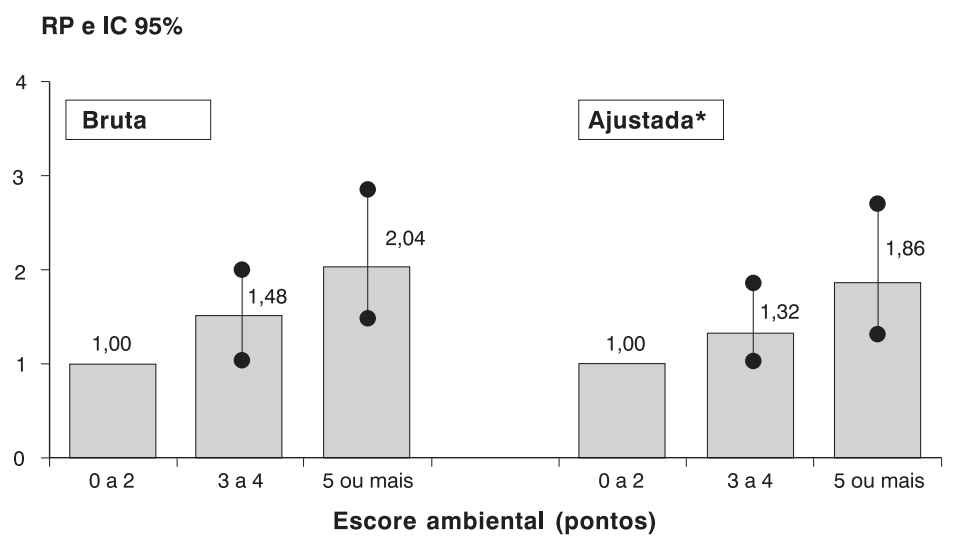

* Ajustada por escolaridade da mãe, renda familiar e idade materna.

Figura 3 - Risco de doença aguda das vias aéreas inferiores, conforme a pontuação do escore ambiental

necessidade de subsidiar um programa específico de atenção às doenças respiratórias nos primeiros anos de vida. Além disso, o critério diagnóstico aplicado apresenta alta sensibilidade e baixa especificidade, o que pode superestimar a ocorrência de doença. Ao excluir as crianças que apresentaram recorrência de sibilância, há uma tendência em aumentar a especificidade, embora não possa ser descartada a inclusão de asmáticos ou sibilantes transitórios. Parece-nos pouco provável, no entanto, que a superestimativa de casos pudesse alterar de forma significativa a elevada prevalência de doença aguda das vias aéreas inferiores encontrada, assim como as associações a ela vinculadas. Por outro lado, ao se considerar essa situação, é preferível preparar o serviço de saúde para atender um caso de broncoespasmo, identificado como doença aguda das vias aéreas inferiores, do que tê-lo como demanda reprimida.

Em relação às variáveis estudadas, escolaridade paterna perdeu a significância após ter sido ajustada para as variáveis demográficas, escolaridade materna e renda familiar. Da mesma forma, a renda familiar perdeu muito poder estatístico após o ajuste, mantendo apenas uma tendência linear significativa. Esse fenômeno pode ser explicado pelo fato de a escolaridade do pai ser, quase sempre, o principal determinante da renda familiar ${ }^{3}$. A escolaridade da mãe, no entanto, manteve a significância estatística, o que vem a reforçar a idéia de que esta seja o mais importante determinante socioeconômico das condições de saúde e de doença das crianças ${ }^{4}$. Como em outros estudos, encontramos um efeito protetor para os filhos de mães mais velhas, mostrando que estas parecem oferecer melhor atenção à saúde infantil ${ }^{20,21}$.

Como em diversos estudos anteriores, aglomeração apareceu como um importante fator de risco para doença respiratória aguda ${ }^{20,22,23}$. Ambos, alto índice de pessoas por cômodo na residência e alto índice de pessoas por quarto, estiveram associados com doença aguda das vias aéreas inferiores na análise bruta. $\mathrm{O}$ índice pessoa por quarto mostrou redução do efeito quando ajustado pelos fatores socioeconômicos, idade materna e demais variáveis ambientais, mas permaneceu significativamente associado. Tais resultados indicam que a aglomeração tem muito de seu efeito confundido por aqueles fatores, no entanto exerce algum efeito independente, como fator de risco para doenças respiratórias agudas, em especial quanto ao número de pessoas que compartilham o quarto de dormir com as crianças.

Três aspectos merecem ser salientados quanto aos achados sobre tabagismo neste estudo. O primeiro refere-se à alta prevalência do hábito de fumar nessa população, especialmente das mães, cujo índice está bem acima da média internacional para mulheres, que é de $29 \%^{8}$. O segundo refere-se ao risco apresentado pelo fumo materno. $\mathrm{Na}$ análise multivariada, após o controle para idade materna, outras variáveis ambientais e nível socioeconômico, o tabagismo materno permaneceu estatisticamente significativo, sem grandes alterações nos valores das razões de prevalência. Esses achados vêm reforçar o risco já bem descrito na literatura sobre a influência do fumo passivo, especialmente da mãe, sobre a saúde respiratória das crianças ${ }^{24,25}$. Quanto ao hábito de fumar durante a gravidez, foi encontrada uma associação significativa para tendência linear, em relação ao número de cigarros fumados, na análise bivariada. Porém, quando ajustada, essa variável perdeu a significância estatística, embora não tenha havido redução importante nas razões de prevalência. A despeito desse achado, esse possível fator de risco necessita de mais investiga- 
ção, especialmente porque não existem estudos relacionados com doença aguda das vias aéreas inferiores. Apenas alguns trabalhos sobre asma e sibilância transitória incluíram essa variável em suas análises, sugerindo existir associação significativa entre o fumo durante a gravidez e a presença de sibilos em crianças menores de cinco anos ${ }^{9,26}$.

Como demonstrado em outros estudos, as condições de moradia desfavoráveis representam um importante fator de risco para doença respiratória ${ }^{3,22,27}$. Os estudos sobre doenças respiratórias agudas têm analisado esses fatores de diversas formas, $\mathrm{e}$ - na maioria das vezes - de forma não muito clara ${ }^{21,27-29}$. A associação direta, estatisticamente significativa, entre o ambiente desfavorável, medido através do número de pontos determinados pelo escore ambiental utilizado, e doença aguda das vias aéreas inferiores permite afirmar que quanto piores as condições do ambiente, maior é a presença de doença respiratória nas crianças.

Os dados deste estudo mostram que há necessidade de melhorar a distribuição de renda e de garantir amplo acesso à escola, com especial atenção à educação materna. Com essas medidas, muitos fatores de risco para doenças respiratórias seriam minimizados, através de melhoria das condições de moradia, com a diminuição da aglomeração familiar, evitando a presença de animais domésticos dentro da casa, e, principalmente, pela redução do hábito de fumar, especialmente entre as mães.

Este trabalho contribui para a melhoria dos serviços oferecidos à população, por proporcionar uma melhor compreensão dos mecanismos causais das doenças respiratórias e por fundamentar a implementação de programas específicos para o seu controle.

\section{Referências bibliográficas}

1. Schmunis G. Prólogo. In: Benguigui Y, editor. Investigações operacionais sobre o controle das infecções respiratórias agudas (IRA). Washington, DC:OPAS; 1997.

2. Benguigui Y. Controle das infecções respiratórias agudas: implementação, acompanhamento e avaliação. Washington, DC: OPAS; 1997.p.2:1-15.

3. Victora CG. Factores de riesgo en las IRA bajas. In: Benguigui Y, Lópes-Antuñano FJ, Yunes J, editores. Infecciones respiratorias en niños. Washington: OPAS; 1996.p.45-63.

4. Victora CG, Barros FC, Vaughan JP. Epidemiologia da desigualdade: um estudo longitudinal de 6.000 crianças brasileiras. $2^{\text {a }}$ ed. São Paulo: Hucitec; 1988.

5. World Health Organization. Rural energy utilization and the health of children. Geneva: WHO; 1985.

6. Pandey MR, Smith KR, Boleij JSM, Wafula EM. Indoor air pollution in developing countries and acute respiratory infections in children. Lancet 1989;1:427-9.

7. Phelan PD, Olinsky A, Robertson CF. Respiratory illness in children. $4^{a}$ ed. Australia: Blackwell Scientific Publication; 1994.p.27-51.
8. American Academy of Pediatrics. Environmental tobacco smoke: a hazard to children - Committee on Environmental Health. Pediatrics 1997;99:639-42.

9. Gergen PJ, Fowler JA, Maurer KR, Davis WW, Overpeck MD. The burden of environmental tobacco smoke exposure on the respiratory health of children 2 months through 5 years of age in the United States: Third National Health and Nutrition Examination Survey, 1988 to 1994. Pediatrics 1998;101(2):E8.

10. Instituto Brasileiro de Geografia e Estatística. Anuário Estatístico. Rio de Janeiro: IBGE; 1997.

11. Dean AG, Dean JA, Coulombier D. Epi Info, Version 6.02: A word processing, database and statistics program for epidemiology on microcomputers. Atlanta (GA): Center of Disease Control and Prevention; 1994.

12. Smith PG, Day NE. The design of case-control studies: the influence of confouding and interactions effects. Int J Epidemiol 1984;13:356-65.

13. Ferris BG. Epidemiology Standardization Project. Am Rev Respir Dis $1978 ; 118: 1-120$.

14. World Health Organization. Programme for control of acute respiratory infections: definitions of clinical signs and symptoms. Interim Programme Report - Draft 1988. Geneva: WHO; 1988.

15. Samet JM, Cushing AH, Lambert WE, Hunt WC, McLaren LC, Young SA, et al. Comparability of parent reports of respiratory illnesses with clinical diagnoses in infants. Am Rev Respir Dis 1993;148:441-6.

16. Norussis M. SPSS for Windows, Version 8.0. Chicago: SPSS Inc., 1998.

17. Rothman KJ. Modern Epidemiology. Boston(MA): Little, Brown and Co., 1986.p.285-310.

18. Mickey RM, Greenland S. The impact of confounder selection criteria on effect estimation. Am J Epidemiol 1989;129:125-37.

19. Victora CG, Huttly SR, Fuchs SC, Olinto MTA. The role of conceptual frameworks in epidemiological analysis: A hierarchical approach. Int J Epidemiol 1997;26:224-7.

20. Fonseca WVC. Risk factors for childhood pneumonia among the poor urban in Fortaleza, Brazil: a case-control study [dissertação]. London: London School of Hygiene and Tropical Medicine; 1993.

21. Amaral JJF, Menezes AMB, Halpern R, Victora CG, Barros FC. Prevalência e fatores de risco para infecção respiratória aguda em crianças aos seis meses de vida em Pelotas, RS. In: Benguigui Y. Investigações operacionais sobre o controle das infecções respiratórias agudas (IRA). Washington: OPAS; 1997.p.85-97.

22. Rahman MM, Rahman AM. Prevalence of acute respiratory tract infections and its risk factors in under five children. Bangladesh Med Res Counc Bull 1997;23:47-50.

23. Zaman K, Baqui AH, Yunus M, Sack RB, Bateman OM, Chowdhury HR, et al. Acute respiratory infections in children: a community-based longitudinal study in rural Bangladesh. J Trop Pediatr 1997;43:133-7.

24. Forastière F, Corbo GM, Michelozzi P, Pistelli R, Agabiti N, Brancato $G$, et al. Effects of environment and passive smoking on the respiratory health of children. Int J Epidemiol 1992;21:66-73.

25. Leeder SR, Corkhill R, Irwig LM, Holland WW. Influence of family factors on the incidence of lower respiratory illness during the first year of life. Br J Prev Soc Med 1976;30:203-12.

26. Phelan PD. Asma em crianças e adolescentes. Baillière's Clinical Paediatrics 1995;3: 247-52.

27. Lopez Bravo IM, Sepulveda H, Valdes I. Acute respiratory illnesses in the first 18 months of life. Rev Panam Salud Publica 1997;1:9-17. 
28. Victora CG, Smith PG, Barros FC, Vaughan JP, Fuchs SC. Risk factors for deaths due to respiratory infections among Brazilian infants. Int J Epidemiol 1989;18:918-25.

29. Victora CG, Fuchs SC, Flores JAC, Fonseca W, Kirkwood B. Risk factors for pneumonia among children in a Brazilian metropolitan area. Pediatrics 1994;93:977-85.
Endereço para correspondência:

Dr. Silvio Omar Macedo Prietsch

Rua Dr. Nascimento, 396 - Centro

CEP 96200-300 - Rio Grande, RS

E-mail:prietsch@uol.com.br 